\title{
A Property of Equivalence
}

\author{
Morris Newman \\ Institute for Basic Standards, National Bureau of Standards, Washington, D.C. 20234
}

(February 6, 1974)

\begin{abstract}
It is shown that if $K, A, B$ are nonsingular matrices over a principal ideal ring $R$ such that $K \otimes A$ is equivalent to $K \otimes B$, then $A$ is equivalent to $B$.

Key words: Elementary divisors; equivalence; Kronecker products.
\end{abstract}

Let $R$ be a principal ideal ring. We write $A \tilde{E} B$, if $A$ and $B$ are matrices over $R$ which are equivalent (see [1] for a complete discussion of this topic). The Kronecker product of any two matrices $A$ and $B$ will be denoted by $A \otimes B$.

The following result was suggested by a remark made by W. D. Wallis in his survey paper [2]: Theorem: Suppose that K, A, B are nonsingular matrices over $\mathrm{R}$ such that $\mathrm{K} \otimes \mathrm{A} \widetilde{\mathrm{E}} \mathrm{K} \otimes \mathrm{B}$. Then A $\tilde{\mathrm{E}} \mathrm{B}$.

It is not actually necessary to assume that $A$ and $B$ are nonsingular, but doing so simplifies the exposition.

We first prove the following:

LEMma: Suppose that the sets

$$
\left\{\mathrm{k}_{\mathrm{i}}+\mathrm{a}_{\mathrm{j}} ; 1 \leqslant \mathrm{i} \leqslant \mathrm{r}, 1 \leqslant \mathrm{j} \leqslant \mathrm{s}\right\},\left\{\mathrm{k}_{\mathrm{i}}+\mathrm{b}_{\mathrm{j}} ; 1 \leqslant \mathrm{i} \leqslant \mathrm{r}, 1 \leqslant \mathrm{j} \leqslant \mathrm{s}\right\}
$$

are the same, where $\mathrm{k}_{1}, \mathrm{k}_{2}, \ldots, \mathrm{k}_{\mathrm{r}}, \mathrm{a}_{1}, \mathrm{a}_{2}, \ldots, \mathrm{a}_{\mathrm{s}}, \mathrm{b}_{1}, \mathrm{~b}_{2}, \ldots, \mathrm{b}_{\mathrm{s}}$ are positive integers. Then the sets

$$
\left\{a_{j} ; 1 \leqslant j \leqslant s\right\},\left\{b_{j} ; 1 \leqslant j \leqslant s\right\}
$$

are the same.

Proof: Since the sets (1) are the same, we must have the polynomial identity

$$
\sum_{\substack{1 \leqslant i \leqslant r \\ 1 \leqslant j \leqslant s}} z^{k i+a_{j}}=\sum_{\substack{1 \leq i \leq r \\ 1 \leqslant j \leqslant s}} z^{k i+b_{j}}
$$

Hence

$$
\left\{\sum_{1 \leqslant i \leqslant r} z^{k_{i}}\right\}\left\{\sum_{1 \leqslant j \leqslant s} z^{a_{j}}\right\}=\left\{\sum_{1 \leqslant i \leqslant r} z^{k_{i}}\right\}\left\{\sum_{1 \leqslant j \leqslant s} z^{b_{j}}\right\}, \quad \sum_{1 \leqslant j \leqslant s} z^{a_{j}}=\sum_{1 \leqslant j \leqslant s} z^{b^{j}}
$$

which implies that the sets (2) must be the same. This completes the proof.

AMS Subject Classification: 15A21; $15 \mathrm{~A} 36$. 
We now prove the theorem. Let $\pi$ be any prime of $R$. Let

$$
\pi^{k 1}, \pi^{k 2}, \ldots, \pi^{k r}
$$

be the elementary divisors of $K$ which are powers of $\pi$,

$$
\pi^{a_{1}}, \pi^{a_{2}}, \ldots, \pi^{a_{s}}
$$

the elementary divisors of $A$ which are powers of $\pi$, and

$$
\pi^{b_{1}}, \pi^{b_{2}}, \ldots, \pi^{b_{t}}
$$

the elementary divisors of $B$ which are powers of $\pi$. Then the elementary divisors of $K \otimes A$ which are powers of $\pi$ are

$$
\pi^{k_{i}+a_{j}}, 1 \leqslant i \leqslant r, 1 \leqslant j \leqslant s ;
$$

and the elementary divisors of $\mathrm{K} \otimes \mathrm{B}$ which are powers of $\pi$ are

$$
\pi^{k i+b_{j}, 1 \leqslant i \leqslant r, 1 \leqslant j \leqslant t}
$$

(see [1], chapter 2).

Since $K \otimes A \widetilde{E} K \otimes B,(3)$ and (4) must be the same (so that $s=t$ ) and hence the sets

$$
\left\{k_{i}+a_{j} ; 1 \leqslant i \leqslant r, 1 \leqslant j \leqslant s\right\},\left\{k_{i}+b_{j} ; 1 \leqslant i \leqslant r, 1 \leqslant j \leqslant s\right\}
$$

must be the same. By the Lemma, the sets $\left\{a_{j} ; 1 \leqslant j \leqslant s\right\}$, $\left\{b_{j} ; 1 \leqslant j \leqslant s\right\}$ must also be the same. Hence the sets

$$
\left\{\pi^{a_{j}} ; 1 \leqslant j \leqslant s\right\},\left\{\pi^{b_{j}} ; 1 \leqslant j \leqslant s\right\}
$$

are the same, so that the elementary divisors of $A$ and $B$ which are powers of any fixed prime are the same. It follows that $A$ and $B$ have the same elementary divisors, and hence that $A \tilde{E} B$. This completes the proof.

The same method of proof also shows that if $A, B$ are nonsingular matrices over $R$ such that

$$
A \otimes A \otimes \ldots \otimes A \tilde{E} B \otimes B \otimes \ldots \otimes B
$$

where there are the same number of $A$ 's as there are $B$ 's in the Kronecker products, then $A \widetilde{E} B$.

\section{References}

[1] Newman, Morris, Integral Matrices (Academic Press, New York, 1972).

[2] Wallis, W. D., On the Number of Inequivalent Hadamard Matrices. Proc. Second Manitoba Conference on Numerical Math., 383-401(1972). 